\title{
Saikosaponin-D improves fear memory deficits in ovariectomized rats via the action of estrogen receptor- $\alpha$ in the hippocampus
}

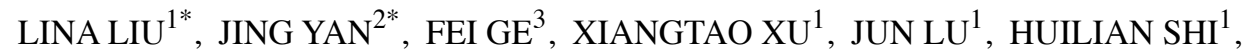 \\ SHUIHONG $\mathrm{LI}^{4}$, YUE ZHAO ${ }^{4}$ and CHANGZHENG ZHANG ${ }^{5}$ \\ ${ }^{1}$ Department of Hepatology, The Affiliated Hospital of Nanjing University of Chinese Medicine, Nanjing, Jiangsu 210029; \\ ${ }^{2}$ Key Laboratory for Metabolic Diseases in Chinese Medicine, First Clinical Medical College, Nanjing University of Chinese \\ Medicine, Nanjing, Jiangsu 210023; ${ }^{3}$ Department of Gastroenterology, Haian Hospital of Traditional Chinese Medicine \\ Affiliated to Nanjing University of Chinese Medicine, Haian, Jiangsu 226600; ${ }^{4}$ Department of Psychiatry and Imageology, \\ Central People's Hospital of Zhanjiang Affiliated to Guangdong Medical University; ${ }^{5}$ Department of Psychology and \\ Key Laboratory of Psychological Assessment and Rehabilitation for Exceptional Children, \\ Lingnan Normal University, Zhanjiang, Guangdong 524048, P.R. China
}

Received September 9, 2018; Accepted April 25, 2019

DOI: $10.3892 / \mathrm{mmr} .2019 .10232$

\begin{abstract}
Saikosaponin-D (SSD), which is the main bioactive component in the traditional Chinese medicine Chai $\mathrm{Hu}$ (Bupleurum falcatum L), possesses estrogen-like properties and is widely used in treating estrogen-related neurological disorders. The current study aimed to investigate the protective effects of SSD on the fear memory deficit in ovariectomized (OVX) rats and the potential underlying mechanism. SSD treatment significantly prolonged freezing time in OVX rats in a manner similar to that of estradiol (E2), whereas this effect was markedly suppressed by co-administration of ICI182780, a non-selective estrogen receptor (ER) inhibitor. The expression of ER $\alpha$ in the hippocampus of OVX rats was significantly elevated by SSD; however, ER $\beta$ expression and E2 synthesis were not markedly affected by SSD treatment. Collectively, this study demonstrated that SSD-mediated fear memory improvement in OVX rats may be attributed not to E2 levels or ER $\beta$ activity, but to ER $\alpha$ activation in the hippocampus.
\end{abstract}

\section{Introduction}

Epidemiological studies have revealed that menopausal women have a greater risk of developing dysmnesia, and the prevalence of

Correspondence to: Dr Changzheng Zhang, Department of Psychology and Key Laboratory of Psychological Assessment and Rehabilitation for Exceptional Children, Lingnan Normal University, 29 Cunjin Road, Chikan, Zhanjiang, Guangdong 524048, P.R. China E-mail: neurobiologyzhang@yahoo.com

\section{${ }^{*}$ Contributed equally}

Key words: saikosaponin-D, ovariectomy, fear memory, estrogen, estrogen receptor
Alzheimer's disease in women is twice as high as that in men (1). This sex difference is correlated with lower levels of ovarian hormones, particularly the potent estrogen hormone estradiol (E2) (2). The specific mechanism underlying E2-mediated dementia is not clearly understood, although substantial evidence indicates that an estrogen deficit leads to glutamate toxicity, causes amyloid- $\beta$ deposition, affects neural plasticity, perturbs neurotrophic activities, evokes neuroinflammation, and accelerates neuronal apoptosis in the brain in menopausal women and ovariectomized (OVX) animals (3-6). Notably, estrogen replacement therapy has been frequently used in young women with primary ovarian insufficiency and in postmenopausal women for preventing dementia or cognitive disorders (7-9).

Phytoestrogens are plant-derived estrogen compounds, which are effective substitutes for clinical estrogen usage $(10,11)$. The traditional Chinese medicine, Xiaoyao San, contains Chai $\mathrm{Hu}$ (Bupleurum falcatum L), which is widely used to ameliorate the postmenopausal syndrome and memory impairment under chronic psychological stress (12-14). The principal bioactive ingredients of Chai $\mathrm{Hu}$ are saikosaponins, which comprise three major subtypes: Saikosaponin-A, saikosaponin-C and saikosaponin-D (SSD) aglycones $(15,16)$. Among these subtypes, SSD (Fig. 1) has attracted interest in medical research and clinical trials, due to its potent bioavailability (17). For example, SSD has been reported to significantly ameliorate postmenopausal syndrome and depressive behaviors $(18,19)$.

The hippocampus is pivotal for memory acquisition, consolidation and extinction. In addition, estrogen action in the hippocampus, particularly in the CA1 region, has important roles in the memory process (20). The present study aimed to determine the modulatory role of SSD in fear memory deficit in OVX rats, as well as the underlying mechanism relating to estrogen action in the hippocampal CA1 region. The present findings may provide a promising therapeutic strategy for the treatment of memory deficits, particularly in postmenopausal women. 


\section{Materials and methods}

Animals. In total, 84 adult female Sprague-Dawley rats (weight, 180-220 g; age, 6-7 weeks) were obtained from Beijing Vital River Laboratory Animal Technology Co., Ltd. All rats were individually housed in cages under a 12 -h light/dark cycle (lights on at 07:00 a.m.) at a temperature of $22 \pm 2^{\circ} \mathrm{C}$ and a humidity of $50-65 \%$. The rats had free access to water and rodent chow. The experimental procedures were approved by The Institutional Committee on The Care and Use of Animals of Nanjing University of Chinese Medicine. Every effort was made to minimize the number of animals used and their suffering. The experimental procedure is presented in Fig. 2.

Implantation of the infusion cannula. The rats were anesthetized with sodium pentobarbital $(40 \mathrm{mg} / \mathrm{kg}$, intraperitoneal injection; cat. no. 20021216; Sinopharm Chemical Reagent Co., Ltd.) and were then mounted on a stereotaxic frame (RWD Life Science Co., Ltd.) for implantation of the infusion cannula [guide cannula: Inner diameter (ID) $=0.38 \mathrm{~mm}$, outer diameter $(\mathrm{OD})=0.56 \mathrm{~mm}$; internal cannula: $\mathrm{ID}=0.36 \mathrm{~mm}, \mathrm{OD}=0.20 \mathrm{~mm}$; RWD Life Science Co.,Ltd.]. The cannula was implanted toward the right lateral ventricle $(\mathrm{A},-0.8 ; \mathrm{L}, 1.5 ; \mathrm{H}, 4.0)$ according to a rat brain atlas (21). The cannula was then anchored to the skull with dental cement and skull screws. An osmotic mini pump was implanted subcutaneously (s.c.) on the back and connected by a tube to the infusion cannula. After the surgery, rats were allowed to recover for at least 7 days in their cages.

Ovariectomy. Animals were anesthetized with sodium pentobarbital, and the bilateral ovaries were excised through flank incisions $(1.5 \mathrm{~cm}$ inferior to the palpated rib cage; $1-2 \mathrm{~cm}$ from the spine). Sham surgery was performed similarly to the ovariectomy procedure but without ovary removal. After the surgery, rats were placed in their cages and allowed to recover for 7 days.

Drug preparation and drug treatment. SSD (purity $\geq 95 \%$; cat. no. S9321; Sigma-Aldrich; Merck KGaA) was first dissolved in 12\% Tween-80 (cat. no. P4780; Sigma-Aldrich; Merck KGaA) and then diluted with $0.9 \%$ saline to a final Tween concentration of 0.5\%. E2 (cat. no. E8875; Sigma-Aldrich; Merck KGaA) was dissolved in sesame oil. The non-selective estrogen receptor (ER) inhibitor ICI182780 (cat. no. 1047/1; Tocris Bioscience) was first dissolved in dimethyl sulfoxide (10 mM; cat. no. d103273; Shanghai Aladdin Biochemical Technology Co., Ltd.) and then diluted to $0.1 \%$ by volume in artificial cerebrospinal fluid (aCSF). The composition of the aCSF was: $\mathrm{NaCl} 124.0 \mathrm{mM}, \mathrm{KCl} 3.0 \mathrm{mM}, \mathrm{NaHCO}_{3}$ $26.0 \mathrm{mM}, \mathrm{MgCl}_{2} \cdot 6 \mathrm{H}_{2} \mathrm{O} 1.2 \mathrm{mM}, \mathrm{NaH}_{2} \mathrm{PO}_{4} \cdot 2 \mathrm{H}_{2} \mathrm{O} 1.2 \mathrm{mM}$, $\mathrm{C}_{6} \mathrm{H}_{12} \mathrm{O}_{6} 10.0 \mathrm{mM}, \mathrm{CaCl}_{2} 2.0 \mathrm{mM}$. All chemical reagents were purchased from Sigma-Aldrich (Merck KGaA).

OVX rats were randomly divided into six subgroups ( $\mathrm{n}=12$ /subgroup): Saline subgroup, $0.9 \% \mathrm{NaCl}$ containing $0.5 \%$ Tween- 80 was administered via intragastric gavage (i.g.) to the rats at the same concentration as in the other groups for 5 weeks; O-E2 subgroup, $2 \mu \mathrm{g} / \mathrm{kg}$ E2 (s.c.) was administered for 5 weeks; O-SSD 1.8 subgroup, $1.8 \mathrm{mg} / \mathrm{kg}$ SSD (i.g.) was administered for 5 weeks; O-SSD 0.9 subgroup, $0.9 \mathrm{mg} / \mathrm{kg} \mathrm{SSD}$ (i.g.) was administered for 5 weeks; O-SSD $1.8-\mathrm{ICI}, 1.8 \mathrm{mg} / \mathrm{kg} \mathrm{SSD}$ was administered for 5 weeks, with

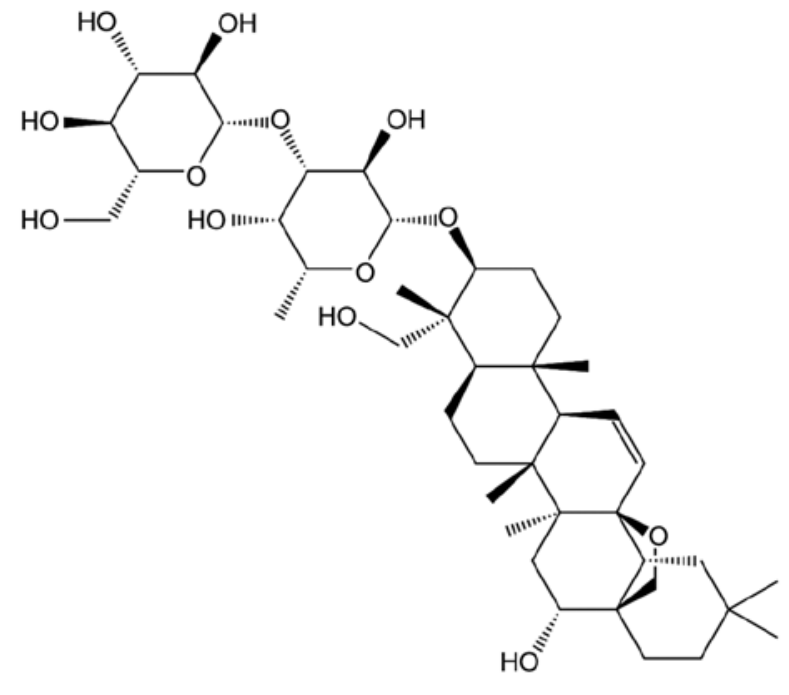

Saikosaponin D

Figure 1. Molecular structure of saikosaponin-D.

$500 \mu \mathrm{g} /$ day ICI182780 (intracerebroventricular injection) co-administered for the final week; and O-SSD 0.9-ICI subgroup, $0.9 \mathrm{mg} / \mathrm{kg}$ SSD was administered for 5 weeks, with ICI182780 co-administered for the final week. The dosages of SSD (0.9 and $1.8 \mathrm{mg} / \mathrm{kg}$ ) used in the current study were based on previous reports, on the basis of sufficient behavioral discrimination and an absence of significant side effects $(19,22,23)$. Rats in the sham group $(n=12)$ underwent no drug treatment. The experimental procedure is shown in Fig. 2. All drugs were administered with a comparable volume once per day at 8:00 a.m.

Contextual fear conditioning test. Fear conditioning tests were performed in a chamber composed of a plexiglas box $(27 \times 31 \times 36 \mathrm{~cm})$ with stainless steel grids (diameter, $5 \mathrm{~mm}$; pitch, $15.7 \mathrm{~mm}$ ) on the floor (Coulbourn Instruments). Each rat was placed in the chamber and subjected to a 2-min adaptation period followed by a 2-min preconditioning phase (without any stimulation) in which the freezing time was measured. During the conditioning period, a tone $(80 \mathrm{~dB})$ was presented as the conditioned stimulus for $20 \mathrm{sec}$, and then a foot shock $(0.5 \mathrm{~mA})$ was delivered as an unconditioned stimulus during the last $2 \mathrm{sec}$ of the tone stimulus. After 10 tone-shock pairs with $60 \mathrm{sec}$ interstimulus intervals (all animals exhibited $>12 \mathrm{sec}$ freezing in a 2-min period), the rats were returned to their cages. Context-dependent tests were performed 1 and $24 \mathrm{~h}$ after the conditioning, in which the freezing time was measured in the same contextual conditions, but without any stimulation, for $3 \mathrm{~min}$. Freezing time $(\%)=$ freezing time/total measurement time (24).

ELISA and western blot analysis. Nine rats randomly selected from each group were sacrificed on the day after behavioral tests under anesthesia at 8:00-9:00 a.m. The skulls were then dissected, and the bilateral hippocampi were quickly removed and placed on ice. The hippocampal CA1 areas were rapidly extracted and cut into $\sim 1 \times 1 \times 0.5 \mathrm{~mm}^{3}$ sections according to the brain atlas (21); these sections were immediately frozen in liquid nitrogen. 


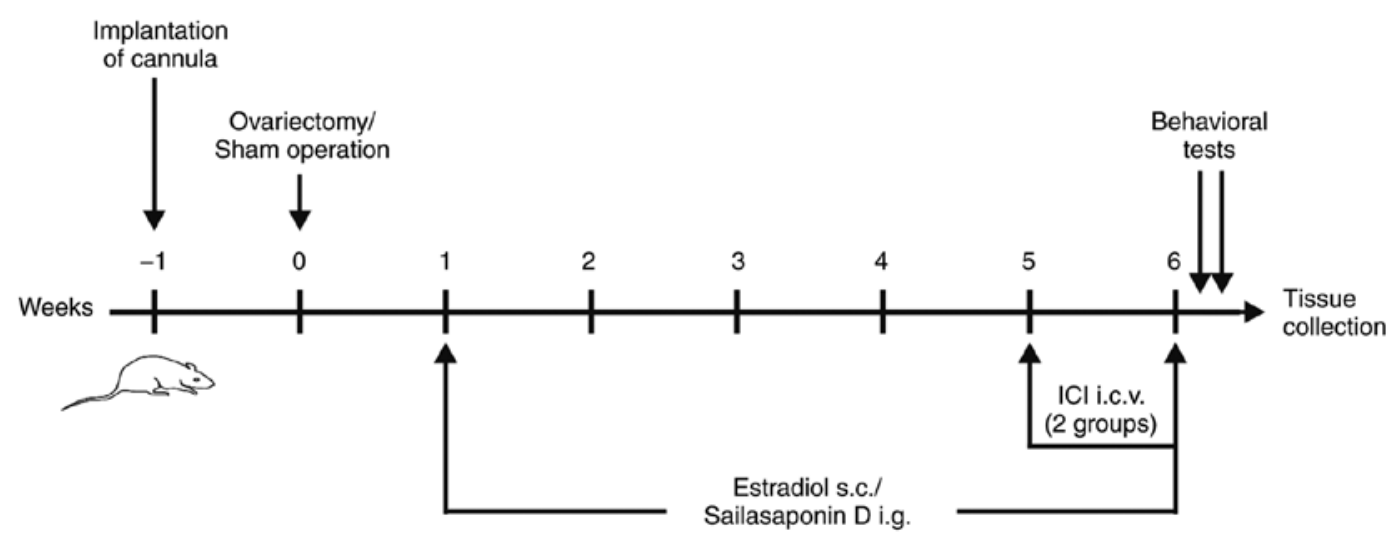

Figure 2. Experimental protocol. Behavioral tests were performed 1 and $24 \mathrm{~h}$ after the last drug administration, and tissues were collected the next day. i.c.v., intracerebroventricular; ICI, ICI182780; i.g., intragastric gavage; s.c., subcutaneous.

Samples from six rats in each group were analyzed by ELISA (cat. no. KGE014; R\&D Systems, Inc.), in order to determine the content of $\mathrm{E} 2$ in the hippocampus, according to the manufacturer's protocol. Absorbance was measured at $450 \mathrm{~nm}$ with a microplate reader.

Samples from the remaining three rats in each group were used for western blot analysis. Tissue proteins were extracted with 5\% RIPA lysis buffer (cat. no. P0013B; Beyotime Institute of Biotechnology), and centrifuged at 13,500 $\mathrm{x}$ g for $15 \mathrm{~min}$ at $4^{\circ} \mathrm{C}$, and the protein concentration was determined using a Pierce bicinchoninic protein assay kit (cat. no. 23223; Thermo Fisher Scientific Inc.). Equal amounts of protein (30 $\mu \mathrm{g} / \mathrm{lane})$ were separated by $7.5-12 \%$ SDS-PAGE (cat. nos. 1610171TA and 1610175TA; Bio-Rad Laboratories, Inc.) and transferred to PVDF membranes (cat. no. IPVH00010; EMD Millipore). The membranes were incubated with TBS containing $0.05 \%$ Tween-20 (TBS-T; cat. no. SRE0031; Sigma-Aldrich; Merck $\mathrm{KGaA}$ ) and $5 \%$ non-fat dry milk for $2 \mathrm{~h}$ at room temperature to block nonspecific binding and were immunoblotted at $4^{\circ} \mathrm{C}$ overnight with the following primary antibodies: Rabbit anti-ER $\alpha$ (1:1,000, cat. no. 21244-1-AP; Proteintech Group, Inc.), rabbit anti-ER $\beta$, (1:1,000, cat. no. 14007-1-AP; Proteintech Group, Inc.), rabbit anti-GAPDH (1:1,000, cat. no. AP0063; Bioworld Technology, Inc.). After being washed by TBS-T, the membranes were incubated with a horseradish peroxidase-conjugated secondary antibody goat anti-rabbit immunoglobulin G (1:5,000, cat. no. BS13278; Bioworld Technology, Inc.) at room temperature for $2 \mathrm{~h}$. Subsequently, the membranes were washed in TBS-T, and were developed with an enhanced chemiluminescence kit (cat. no. NEL104001EA; PerkinElmer, Inc.). Each experiment was performed independently at least three times, and the integrated intensity was measured using Image-Pro-Plus 6.0 (Media Cybernetics, Inc.).

Immunohistochemistry and Nissl staining. In each group, tissues from the remaining three rats were processed for immunohistochemistry, according to our previously described procedure (25). Briefly, the rats were deeply anesthetized and transcardially perfused with $4 \%$ paraformaldehyde (cat. no. AR1068; Wuhan Boster Biological Technology, Ltd.). The brains were extracted, postfixed in $4 \%$ paraformaldehyde at $4^{\circ} \mathrm{C}$ for $24 \mathrm{~h}$ and embedded in paraffin; subsequently, samples were cut into 5- $\mu \mathrm{m}$ sections. The three sections containing the hippocampal CA1 region were incubated with 3\% hydrogen peroxide (cat. no. AR1108; Wuhan Boster Biological Technology, Ltd.) at room temperature for $10 \mathrm{~min}$, $10 \%$ goat serum (cat. no. ab7481; Abcam) at $37^{\circ} \mathrm{C}$ for $30 \mathrm{~min}$. Subsequently, sections were incubated with the primary antibodies rabbit anti-ER $\alpha$ (1:200; cat. no. 21244-1-AP; Proteintech Group, Inc.) or rabbit anti-ER $\beta$ (1:200, cat. no. 14007-1-AP; Proteintech Group, Inc.) at $4{ }^{\circ} \mathrm{C}$ overnight and the biotinylated secondary antibody goat anti-rabbit $\operatorname{IgG}(1: 500$, cat. no. BS13278; Bioworld Technology, Inc.) were incubated at room temperature for $30 \mathrm{~min}$. The sections were subsequently counterstained with hematoxylin $(0.5 \%$; cat. no. H104302; Shanghai Aladdin Biochemical Technology Co., Ltd.) at room temperature for $3 \mathrm{~min}$.

The adjacent slices were selected for Nissl staining ( $0.5 \%$ thionine; cat. no. 78338-22-4; Sigma-Aldrich; Merck KGaA; $37^{\circ} \mathrm{C}, 10 \mathrm{~min}$ ), and micrographs of the hippocampal CA1 region were captured under a light microscope (Axio Vert A1; Carl Zeiss AG). Nissl-labeled neurons were calculated from two or three random fields per slice, and technicians blinded to the samples manually made the measurements. Briefly, in each Nissl-stained slide (magnification, $x 400$ ) the number of neurons was counted in a calibrator $(125 \times 125 \mu \mathrm{m})$ and the density (cells $/ \mathrm{mm}^{2}$ ) was subsequently calculated. The criterion for acceptance as a neuron was clear staining of a soma and a nucleus, which were distinctly differentiated from their backgrounds (25).

Statistical analysis. Data are expressed as the means \pm SEM. The significance of the differences between groups was evaluated by one-way analysis of variance (ANOVA) followed by Fisher's least significant difference post hoc test using SPSS version 16.0 (SPSS Inc.). P $<0.05$ was considered to indicate a statistically significant difference.

\section{Results}

SSD markedly rescues ovariectomy-induced fear memory deficit. Among all groups (sham, O-saline, O-E2, O-SSD 1.8, O-SSD 0.9, O-SSD 1.8-ICI and O-SSD 0.9-ICI), the mean freezing fraction prior to conditioning exhibited no significant differences $\left(\mathrm{F}_{6,77}=0.47, \mathrm{P}=0.65\right.$, one-way ANOVA; 


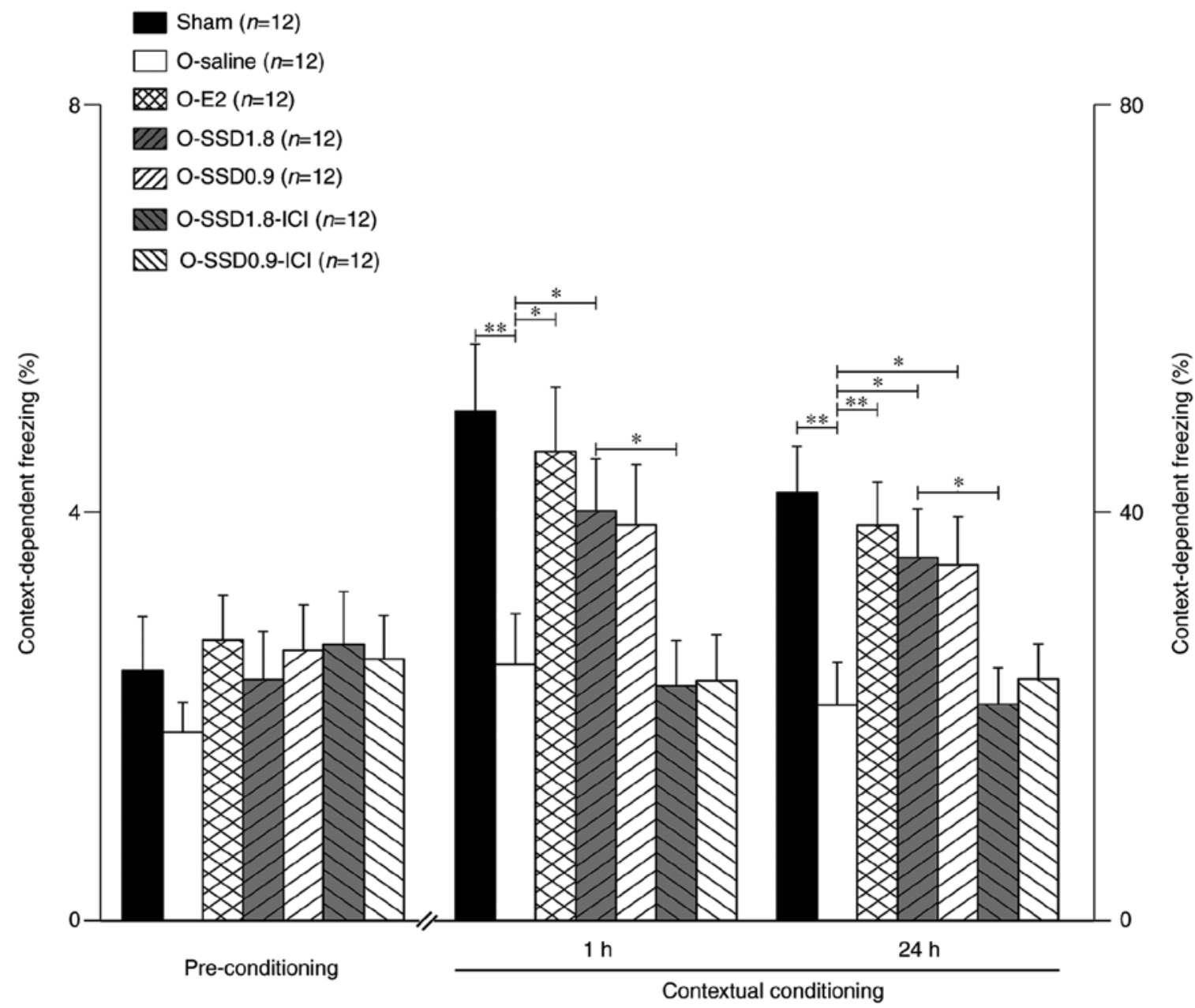

Figure 3. Context-dependent freezing time (\%) among groups. Data are presented as the means $\pm \mathrm{SEM}$. ${ }^{*} \mathrm{P}<0.05 ;{ }^{* *} \mathrm{P}<0.01$, one-way analysis of variance and Fisher's least significant difference post hoc test. E2, estradiol; ICI, ICI182780; O, ovariectomized; SSD, saikosaponin-D.

Fig. 3). Compared with the sham operation group, ovariectomy significantly shortened the freezing time $(\mathrm{P}<0.01$, O-saline vs. sham; Fig. 3); this finding is in agreement with results from a previous study (26). However, the freezing time was markedly prolonged in OVX animals after E2 administration at 1 or $24 \mathrm{~h}$ ( $\mathrm{P}<0.05$ or $\mathrm{P}<0.01, \mathrm{O}-\mathrm{E} 2$ vs. $\mathrm{O}$-saline; Fig. 3$)$, thus indicating that the memory loss in OVX rats was due to an estrogen deficit. Notably, treatment with SSD (SSD 1.8 and SSD 0.9) exerted a similar effect to E2 administration, which lasted for at least $24 \mathrm{~h}$ despite slight attenuation (Fig. 3).

Intracerebroventricular administration of ICI182780 markedly blocked the increase in freezing time in the O-SSD 1.8 group at $1 \mathrm{~h}(\mathrm{P}<0.05 \mathrm{O}-\mathrm{SSD} 1.8$-ICI vs. O-SSD 1.8) and $24 \mathrm{~h}$ $(\mathrm{P}<0.05$, O-SSD 1.8-ICI vs. O-SSD 1.8; Fig. 3), and there was also a clear tendency toward reduced freezing in the O-SSD 0.9-ICI group (Fig. 3). These results indicated that ERs may be involved in SSD-mediated effects.

SSD does not affect E2 levels in the hippocampus of OVX rats. The $\mathrm{E} 2$ levels in the hippocampus were significantly lower in OVX rats compared with in sham-operated animals $(\mathrm{P}<0.01, \mathrm{O}$-saline vs. sham; Fig. 4), whereas this decrease was reversed following $\mathrm{E} 2$ administration $(\mathrm{P}<0.05, \mathrm{O}-\mathrm{E} 2$ vs. O-saline; Fig. 4). However, SSD treatment (O-SSD 1.8 or O-SSD 0.9) exhibited no influence on E2 levels $(\mathrm{P}=0.46$

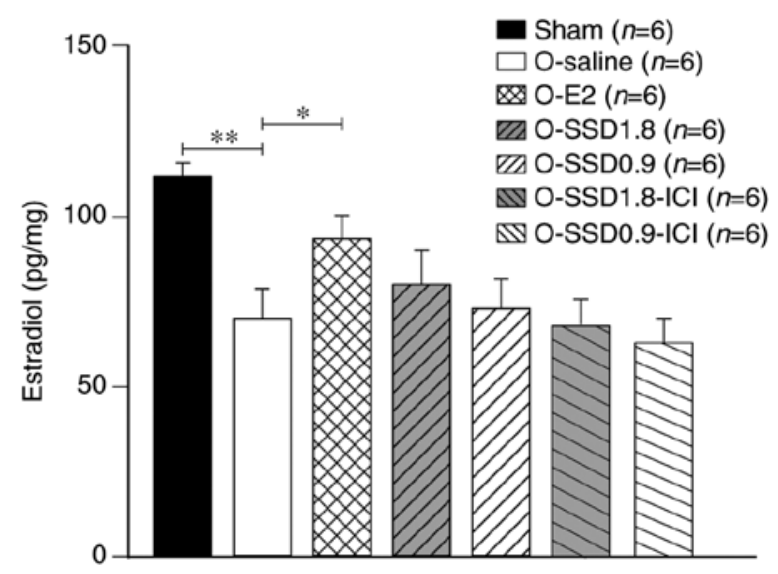

Figure 4. E2 levels in the hippocampal CA1 region among the groups. E2 levels in the hippocampus were measured with an E2 ELISA kit. Data are presented as the means $\pm \mathrm{SEM}$. ${ }^{*} \mathrm{P}<0.05 ;{ }^{* *} \mathrm{P}<0.01$, one-way analysis of variance and Fisher's least significant difference post hoc test. E2, estradiol; ICI, ICI182780; O, ovariectomized; SSD, saikosaponin-D.

and $\mathrm{P}=0.82$, respectively, for O-SSD 1.8 or O-SSD 0.9 vs. O-saline; Fig. 4). In addition, the ER inhibitor ICI182780 did not influence E2 levels ( $\mathrm{P}=0.22$, O-SSD 1.8-ICI vs. O-SSD 1.8; $\mathrm{P}=0.59$, O-SSD 0.9-ICI vs. O-SSD 0.9; Fig. 4). These 

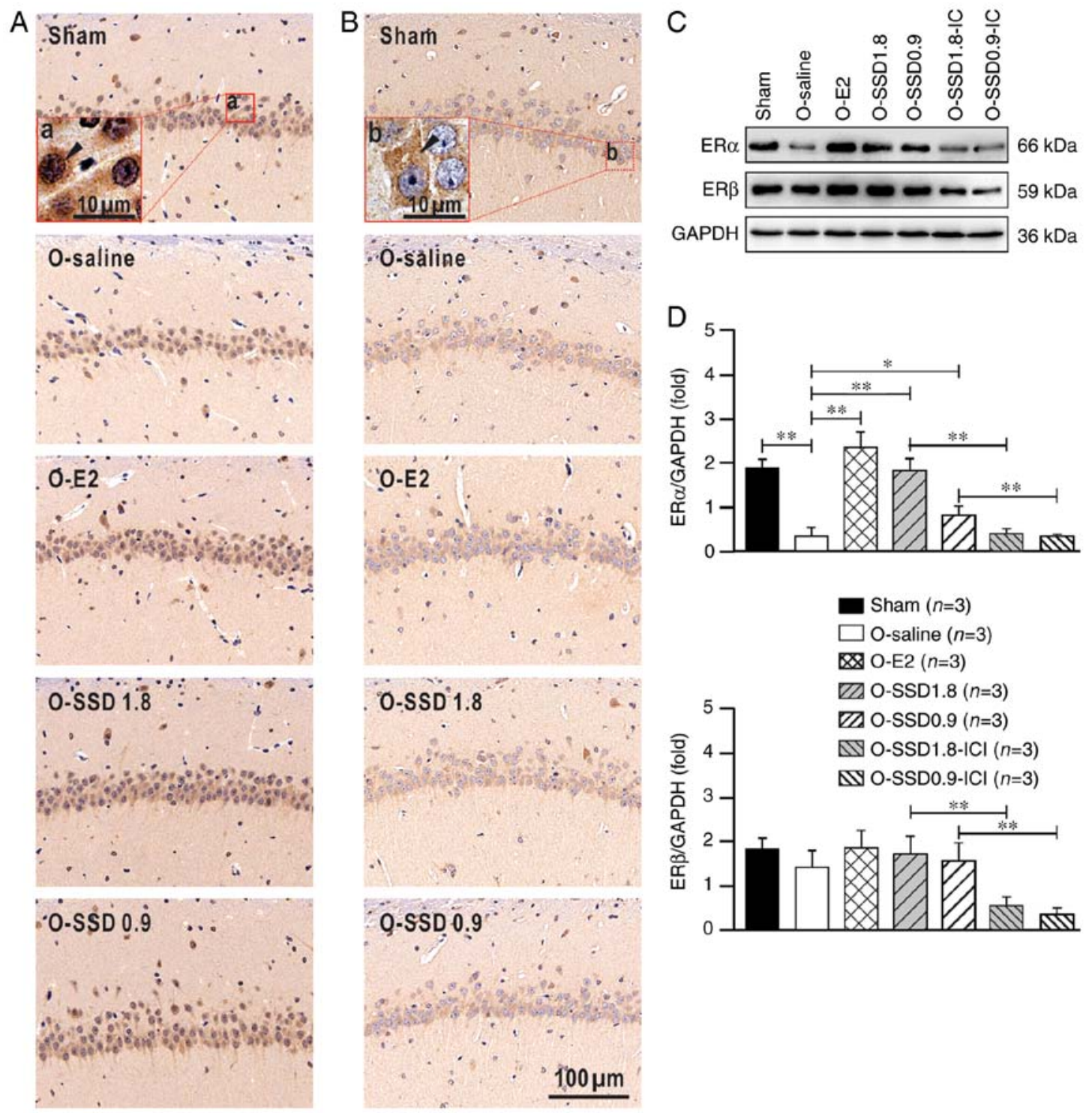

Figure 5. ER $\alpha$ and ER $\beta$ expression in the hippocampal CA1 region among groups. (A and B) Immunohistochemical staining. Sections demonstrated that in the hippocampal CA1 region, ER $\alpha$ was present in extranuclear and nuclear sites, whereas ER $\beta$ was present in extranuclear sites. (a) The red box represents a higher magnification, and the black triangle indicates an ER $\alpha$-positive neuron; (b) the red box represents a higher magnification, and the black triangle indicates an ER $\beta$ positive neuron. (C and D) Western blot analysis for semi-quantitative analysis of ER $\alpha$ and ER $\beta$ expression, whose bands were 66 and $59 \mathrm{kDa}$, respectively. The optical density of ER $\alpha$ or ER $\beta$ was normalized to GAPDH. Data are presented as the means $\pm \mathrm{SEM}$. " $\mathrm{P}<0.05$; ${ }^{* *} \mathrm{P}<0.01$, one-way analysis of variance and Fisher's least significant difference post hoc test. Scale bars: (A and B) $100 \mu \mathrm{m}$; (a and b) $10 \mu \mathrm{m}$. E2, estradiol; ER, estrogen receptor; ICI, ICI182780; O, ovariectomized; SSD, saikosaponin-D.

results suggested that the SSD-mediated improvement in memory deficit in OVX rats may not be attributed to E2 alterations in the hippocampus.

SSD enhances hippocampal expression of ER $\alpha$, but not $E R \beta$, in OVX rats. As shown in Fig. 5, ER $\alpha$ and ER $\beta$ were widely expressed in hippocampal neurons; this has also been reported by previous studies (27-29). ER $\alpha$ was present in both the extranuclear (cytoplasmic regions) and intranuclear sites of the neurons, with prominent extranuclear expression (Fig. 5Aa). In addition, ER $\beta$ was mainly expressed in the extranuclear sites (Fig. 5Bb), which is in line with previous reports (30-32). The staining intensities for ERs exhibited obvious differences among the groups. Western blotting was conducted for semi-quantitative analysis of ER expression (Fig. 5C). ER $\alpha$ exhibited a significant downregulation in the hippocampus of
OVX rats $(\mathrm{P}<0.01$, O-saline vs. sham; Fig. 5D), whereas E2 supplementation substantially reversed this downregulation ( $\mathrm{P}<0.01, \mathrm{O}-\mathrm{E} 2$ vs. O-saline; Fig. 5D). Notably, ER $\alpha$ expression in the hippocampus was also significantly enhanced by SSD treatment in the O-SSD $1.8(\mathrm{P}<0.01, \mathrm{O}-\mathrm{SSD} 1.8$ vs. O-saline; Fig. 5D) and O-SSD 0.9 groups $(\mathrm{P}<0.05$, O-SSD 0.9 vs. O-saline; Fig. 5D) of OVX rats. Conversely, ER $\beta$ expression exhibited no significant differences among these groups (sham, O-saline, O-E2, O-SSD 1.8 and O-SSD 0.9) by one-way ANOVA $\left(\mathrm{F}_{4,10}=0.75, \mathrm{P}=0.58\right.$; Fig. $\left.5 \mathrm{D}\right)$. These findings indicated that SSD activated ER $\alpha$ expression in the hippocampus of OVX rats. Furthermore, ER $\alpha$ and ER $\beta$ expression in the O-SSD groups was markedly inhibited by ICI182780 intervention $(\mathrm{P}<0.01$, O-SSD 1.8-ICI vs. O-SSD 1.8 and O-SSD 0.9 -ICI vs. O-SSD 0.9; Fig. 5D), confirming that the inhibitor ICI182780 had suppressive effects on ER $\alpha$ and ER $\beta$. 

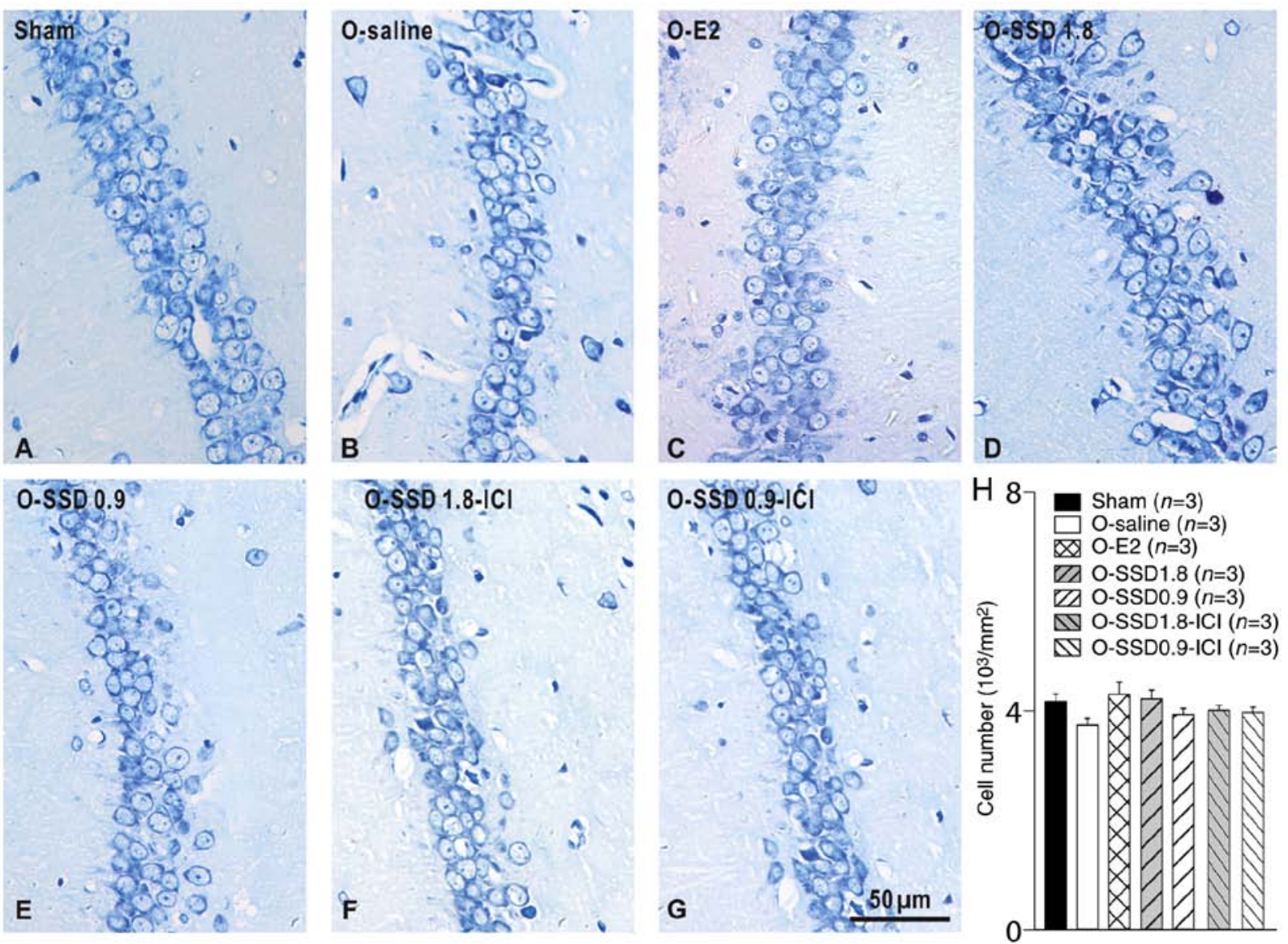

Figure 6. Neuron numbers in the hippocampal CA1 region among the groups. (A-G) Micrographs of Nissl-labeled slices. (H) Histogram of the neuron number. There was no significant difference in neuron number among the groups. Scale bars: 50 $\mu \mathrm{m}$. E2, estradiol; ER, estrogen receptor; ICI, ICI182780; O, ovariectomized; SSD, saikosaponin-D.

SSD does not affect the number of neurons in the hippocampus of OVX rats. Given that neuron number conclusively influences neural functioning in the nervous system, this study aimed to determine whether SSD-induced behavioral improvement in OVX rats may be associated with changes in neuron number. As shown in Fig. 6A-H, the number of neurons in the hippocampus exhibited no significant alterations among the groups (sham, O-saline, O-E2, O-SSD 1.8, O-SSD 0.9, O-SSD 1.8-ICI and O-SSD 0.9-ICI; $\mathrm{F}_{6,119}=1.429, \mathrm{P}=0.209$; Fig. $6 \mathrm{H}$ ), thus suggesting that SSD-mediated improvements in memory deficits in OVX rats were not attributed to changes in the number of neurons.

\section{Discussion}

Estrogen serves important roles in the central nervous system and is involved in various processes, including neuronal differentiation, synaptic formation and neural repair (33-35). Accordingly, estrogen deficits lead to substantial neural malfunction, particularly cognitive impairment $(36,37)$. The hippocampus is a crucial center for cognitive processes, which is extensively modulated by estrogen; furthermore, estrogen disorders often lead to cognitive impairment, including fear memory deficits $(38,39)$. In the present study, contextual fear conditioning was used to reveal the hippocampal action of estrogen involved in fear memory.
E2 levels in the hippocampus are markedly decreased in OVX rats, probably due to a local decrease in estrogenic synthesis (40). The present results demonstrated that decreased E2 in the hippocampus was associated with fear memory impairment in OVX rats. Notably, this impairment was reversed by E2 supplementation, in a manner reminiscent of clinical estrogen replacement therapy for menopausal women or OVX rats $(34,36,41,42)$. However, E2 in the hippocampus exerts distinct roles under different conditions; for example, E2 serves an active role at low doses, whereas higher doses exert a suppressive effect on working memory $(43,44)$. Notably, the phytoestrogen SSD has a similar chemical structure and physiological function to E2, and is frequently used as a substitute for estrogen in clinical trials. In the present study, it was revealed that SSD prolonged freezing time in OVX rats in a manner similar to E2. However, the E2-mediated improvement in memory deficit was associated with elevated hormone levels, whereas the SSD-induced improvement was independent of E2 levels in the hippocampus of OVX rats. In addition, SSD did not exert any influence on serum E2 levels in OVX rats (Liu et al, unpublished data), strongly indicating that the SSD-rescued memory deficit in OVX rats does not rely on E2 levels.

Although ERs are expressed in neurons and glial cells, the expression is highly distinct among different brain regions (30). ERs in the hippocampal CA1 area are mainly expressed in 
neurons (27-29). In the current study, immunohistochemistry was performed to discern the cytoplasmic and nuclear distribution of ERs, and western blotting was used to semi-quantify protein expression. The results revealed that $\mathrm{ER} \alpha$ and $\mathrm{ER} \beta$ were expressed in the hippocampus; however, ER $\beta$ expression in the hippocampal CA1 region remained unchanged in OVX rats. This outcome may be associated with elevated serum corticosterone (Liu et al, unpublished data), since E2 deficiency stimulates the hypothalamic-pituitary-adrenal axis to release corticosterone, which subsequently counteracts the decrease in $\operatorname{ER} \beta(45,46)$. In addition, SSD treatment did not affect ER $\beta$ protein expression. Conversely, significant alterations in ER $\alpha$ expression were detected in the hippocampus of OVX rats following SSD treatment. ER $\alpha$ has frequently been reported to be associated with spine structure and/or postsynaptic functions $(47,48)$; for example, selective activation of ER $\alpha$ in OVX mice results in an increased spine density in the hippocampus, which is inversely correlated with memory dysfunction (49). In particular, ICI182780, a non-selective inhibitor of ERs, markedly blocked the memory improvement induced by SSD in OVX rats, thus corroborating a mechanism of SSD-mediated memory restoration via ERs. Previous studies reported that ovariectomy stimulates ER $\alpha$ promoter methylation, which in turn inhibits ER $\alpha$ expression $(47,50)$. Therefore, it may be hypothesized that SSD prevents ER $\alpha$ promoter methylation and induces ER mRNA expression, thus resulting in upregulation of $\mathrm{ER} \alpha$ protein expression in OVX rats. In the present study, ovariectomy did not alter the number of neurons in the hippocampus; this was also the case in a previous study (46). In addition, neither SSD nor E2 supplementation affected neuron number. Therefore, SSD-induced activation of ER $\alpha$ in hippocampal neurons may be a potential mechanism underlying SSD-improved fear deficits in OVX rats.

Despite the novel findings of the present study, several limitations must be solved for further improvement. Firstly, because the amygdala serves a pivotal role in triggering a state of fear, and is also involved in the modulation of memory consolidation (51), a thorough investigation of the neural circuit for SSD-mediated promotion in fear memory deficit is worthy of future consideration. Secondly, although this study revealed that SSD differentially upregulates ER expression in the hippocampus, whether SSD modulates neuronal activities, and whether ER $\alpha$ and ER $\beta$ have different roles in the hippocampus in OVX animals, remains to be determined. Thirdly, the present study, along with various previous reports, suggested that a suitable low dosage of SSD may benefit neurological functions $(19,52)$, whereas higher concentrations of SSD may cause neurotoxicity $(22,53)$; although the different concentrations of SSD activate distinct signal pathways (54), further research is required to clarify the precise mechanisms.

In conclusion, this study demonstrated that the phytoestrogen SSD rescued fear memory deficit in OVX rats. The results suggested that this effect may be mediated through activation of $\mathrm{ER} \alpha$, rather than $\mathrm{ER} \beta$ or $\mathrm{E} 2$, in the hippocampus.

\section{Acknowledgements}

Not applicable.

\section{Funding}

The present study was supported by the Natural Science Foundation of Jiangsu Province (grant nos. BK20140959 and BK20151008); the National Natural Science Foundation of China (grant nos. 81603578 and 81503536); the Natural Science Foundation of Universities in Jiangsu Province (grant no. 15KJB360008); the Key Laboratory Fund of Philosophy and Social Sciences of Universities in Guangdong Province (Psychological Assessment and Rehabilitation for Exceptional Children; grant no. 2017SYSYJ01).

\section{Availability of data and materials}

All data generated or analyzed during the present study are included in this published article.

\section{Authors' contributions}

LL and CZ conceived and designed the study. LL, JY and XX performed the experiments. HS, FG, JL SL and YZ analyzed the data and revised the manuscript. LL, JY and CZ wrote the manuscript. All authors read and approved the final version of the manuscript.

\section{Ethics approval and consent to participate}

All experimental protocols involving animal tissue samples were approved by the Ethics Committee of Nanjing University of Chinese Medicine.

\section{Patient consent for publication}

Not applicable.

\section{Competing interests}

The authors declare that they have no competing interests.

\section{References}

1. Heber LE, Weuve J, Scherr PA and Evans DA: Alzheimer disease in the United States (2010-2050) estimated using the 2010 census. Neurology 80: 1778-1783, 2013.

2. Simpkins JW, Green PS, Gridley KE, Singh M, de Fiebre NC and Rajakumar G: Role of estrogen replacement therapy in memory enhancement and the prevention of neuronal loss associated with Alzheimer's disease. Am J Med 103: S19-S25, 1997.

3. Correia SC, Santos RX, Cardoso S, Carvalho C, Santos MS Oliveira CR and Moreira PI: Effects of estrogen in the brain: Is it a neuroprotective agent in Alzheimer's disease? Cur Aging Sci 3: 113-126, 2010.

4. Pompili A, Arnone B and Gasbarri A: Estrogens and memory in physiological and neuropathological conditions. Psychoneuroendocrino 37: 1379-1396, 2012.

5. Bonomo SM, Rigamonti AE, Giunta M, Galimberti D, Guaita A, Gagliano MG, Müller EE and Cella SG: Menopausal transition: A possible risk factor for brain pathologic events. Neurobiol Aging 30: 71-80, 2009.

6. Karisetty BC, Maitra S, Wahul AB, Musalamadugu A, Khandelwal N, Guntupalli S, Garikapati R, Jhansyrani T, Kumar A and Chakravarty S: Differential effect of chronic stress on mouse hippocampal memory and affective behavior: Role of major ovarian hormones. Behav Brain Res 318: 36-44, 2017.

7. Sullivan SD, Sarrel PM and Nelson LM: Hormone replacement therapy in young women with primary ovarian insufficiency and early menopause. Fertil Steril 106: 1588-1599, 2016. 
8. Osmanovic-Barilar J and Salkovic-Petrisi M: Evaluating the role of hormone therapy in postmenopausal women with Alzheimer's disease. Drug Aging 33: 787-808, 2016.

9. Lewis CE and Wellons MF: Menopausal hormone therapy for primary prevention of chronic disease. JAMA 318: 2187, 2017

10. Kennedy DO and Scholey AB: The psychopharmacology of European herbs with cognition-enhancing properties. Curr Pharm Design 12: 4613-4623, 2006.

11. Atteritano M, Pernice F, Mazzaferro S, Mantuano S, Frisina A D'Anna R, Cannata ML, Bitto A, Squadrito F, Frisina N and Buemi M: Effects of phytoestrogen genistein on cytogenetic biomarkers in postmenopausal women: 1 year randomized, placebo-controlled study. Eur J Pharmacol 589: 22-26, 2008.

12. Yang YH, Chen PC, Wang JD, Lee CH and Lai JN: Prescription pattern of traditional Chinese medicine for climacteric women in Taiwan. Climacteric 12: 541-547, 2009.

13. Lee B, Shim I, Lee H and Hahm DH: Effect of Bupleurum falcatum on the stress-induced impairment of spatial working memory in rats. Biol Pharm Bull 32: 1392-1398, 2009.

14. Chen JX, Ji B, Lu ZL and Hu LS: Effects of chai hu (radix burpleuri) containing formulation on plasma beta-endorphin, epinephrine and dopamine on patients. Am J Chinese Med 33: $737-745,2005$

15. Morinaga O, Zhu S, Tanaka $\mathrm{H}$ and Shoyama Y: Visual detection of saikosaponins by on-membrane immunoassay and estimation of traditional Chinese medicines containing Bupleuri radix. Biochem Bioph Res Commun 346: 687-692, 2006.

16. de Oliveira DR, Zamberlam CR, Rêgo GM, Cavalheiro A, Cerutti JM and Cerutti SM: Effects of a flavonoid-rich fraction on the acquisition and extinction of fear memory: Pharmacological and molecular approaches. Front Behav Neurosci 9: 345, 2016.

17. Aoyagi H, Kobayashi Y, Yamada K, Yokoyama M, Kusakari K and Tanaka H: Efficient production of saikosaponins in Bupleurum falcatum root fragments combined with signal transducers. Appl Microbiol Biot 57: 482-488, 2001.

18. Lin J, Zhu J, Wang Y, Zhang N, Gober HJ, Qiu X, Li D and Wang L: Chinese single herbs and active ingredients for postmenopausal osteoporosis: From preclinical evidence to action mechanism. Biosci Trends 11: 496-506, 2017.

19. Li HY, Zhao YH, Zeng MJ, Fang F, Li M, Qin TT, Ye LY, Li HW, Qu R and Ma SP: Saikosaponin D relieves unpredictable chronic mild stress induce d depressive-like behavior in rats: Involvement of HPA axis and hippocampal neurogenesis. Psychopharmacology (Berl) 234: 3385-3394, 2017.

20. McEwen B, Akama K, Alves S, Brake WG, Bulloch K, Lee S, Li C, Yuen G and Milner TA: Tracking the estrogen receptor in neurons: Implications for estrogen-induced synapse formation. Proc Natl Acad Sci USA 98: 7093-7100, 2001.

21. Paxinos $\mathrm{G}$ and Watson $\mathrm{C}$ : The rat brain in stereotaxic coordinates. Academic Press/Elsevier, Amsterdam; Boston, 2007.

22. Dang SS, Wang BF, Cheng YA, Song P, Liu ZG and Li ZF: Inhibitory effects of saikosaponin-d on CCl4-induced hepatic fibrogenesis in rats. World J Gastroentero 13: 557-563, 2007.

23. Lu XL, He SX, Ren MD, Wang YL, Zhang YX and Liu EQ: Chemopreventive effect of saikosaponin-d on diethylinitrosamine-induced hepatocarcinogenesis: Involvement of CCAAT/enhancer binding protein $\beta$ and cyclooxygenase- 2 . Mol Med Rep 5: 637-644, 2012.

24. Takuma K, Mizoguchi H, Funatsu Y, Hoshina Y, Himeno Y, Fukuzaki E, Kitahara Y, Arai S, Ibi D, Kamei H, et al: Combination of chronic stress and ovariectomy causes conditioned fear memory deficits and hippocampal cholinergic neuronal loss in mice. Neuroscience 207: 261-273, 2012

25. Zhang C, Sun T, Zhou P, Zhu Q and Zhang L: Role of muscarinic acetylcholine receptor- 2 in the cerebellar cortex in cardiovascular modulation in anaesthetized rats. Neurochem Res 41: 804-812, 2016.

26. Mcdermott CM, Liu D, Ade C and Schrader LA: Estradiol replacement enhances fear memory formation, impairs extinction and reduces COMT expression levels in the hippocampus of ovariectomized female mice. Neurobiol Learn Mem 118: 167-177, 2014.

27. Shughrue PJ and Merchenthaler I: Evidence for novel estrogen binding sites in the rat hippocampus. Neuroscience 99: 605-612, 2000 .

28. Loy R, Gerlach JL and Mcewen BS: Autoradiographic localization of estradiol-binding neurons in the rat hippocampal formation and entorhinal cortex. Dev Brain Res 39: 245-251, 1988.
29. Adams MM, Fink SE, Shah RA, Janssen WG, Hayashi S, Milner TA, McEwen BS and Morrison JH: Estrogen and aging affect the subcellular distribution of estrogen receptor-alpha in the hippocampus of female rats. J Neurosci 22: 3608-3614, 2002.

30. Mitterling KL, Spencer JL, Dziedzic N, Shenoy S, McCarthy K, Waters EM, McEwen BS and Milner TA: Cellular and subcellular localization of estrogen and progestin receptor immunoreactivities in the mouse hippocampus. J Comp Neurol 518: 2729-2743, 2010.

31. Milner TA, McEwen BS, Hayashi S, Li CJ, Reagan LP and Alves SE: Ultrastructural evidence that hippocampal alpha estrogen receptors are located at extranuclear sites. J Comp Neurol 429: 355-371, 2001.

32. Milner TA, Ayoola K, Drake CT, Herrick SP, Tabori NE, McEwen BS, Warrier S and Alves SE: Ultrastructural localization of estrogen receptor $\beta$ immunoreactivity in the rat hippocampal formation. J Comp Neurol 491: 81-95, 2005.

33. Heberden C: Sex steroids and neurogenesis. Biochem Pharmacol 141: 56-62, 2017.

34. Hara Y, Waters EM, Mcewen BS and Morrison JH: Estrogenzeffects on cognitive and synaptic health over the lifecourse. Physiol Rev 95: 785-807, 2015.

35. Vierk R, Bayer J, Freitag S, Muhia M, Kutsche K, Wolbers T, Kneussel M,SommerT andRuneGM:Structure-function-behavior relationship in estrogen-induced synaptic plasticity. Horm Behav 74: 139-148, 2015.

36. Herrera AY, Hodis HN, Mack WJ and Mather M: Estradiol therapy after menopause mitigates effects of stress on cortisol and working memory. J Clin Endocrinol Metab 102: 4457-4466, 2017.

37. Foy MR, Baudry M, Diaz BR and Thompson RF: Estrogen and hippocampal plasticity in rodent models. J Alzheimers Dis 15: 589, 2008.

38. Barha CK, Dalton GL and Galea LA: Low doses of 17alpha-estradiol and 17beta-estradiol facilitate, whereas higher doses of estrone and 17alpha- and 17beta-estradiol impair, contextual fear conditioning in adult female rats. Neuropsychopharmacol 35: 547-559, 2010.

39. Hoffman AN, Armstrong CE, Hanna JJ and Conrad CD: Chronic stress, cyclic $17 \beta$-estradiol, and daily handling influences on fear conditioning in the female rat. Neurobiol Learn Mem 94: 422-433, 2010

40. Barker JM and Galea LAM: Sex and regional differences in estradiol content in the prefrontal cortex, amygdala and hippocampus of adult male and female rats. Gen Comp Endocr 164: 77-84, 2009

41. Rodgers SP, Bohacek J and Daniel JM: Transient estradiol exposure during middle age in ovariectomized rats exerts lasting effects on cognitive function and the hippocampus. Endocrinology 151: 1194-1203, 2010.

42. Bean LA, Kumar A, Rani A, Guidi M, Rosario AM, Cruz PE, Golde TE and Foster TC: Re-opening the critical window for estrogen therapy. J Neurosci 35: 16077-16093, 2015.

43. Holmes MM, Wide JK and Galea LA: Low levels of estradiol facilitate, whereas high levels of estradiol impair, working memory performance on the radial arm maze. Behav Neurosci 116: 928-934, 2002

44. Wide JK, Hanratty KJ and Galea LA: High level estradiol impairs and low level estradiol facilitates non-spatial working memory. Behav Brain Res 155: 45-53, 2004.

45. Wei N, Yu Y, Schmidt T, Stanford C and Hong L: Effects of glucocorticoid receptor antagonist, RU486, on the proliferative and differentiation capabilities of bone marrow mesenchymal stromal cells in ovariectomized rats. J Orthop Res 31: 760-767, 2013.

46. van de Stolpe A, Slycke AJ, Reinders MO, Zomer AW, Goodenough S, Behl C, Seasholtz AF and van der Saag PT: Estrogen receptor (ER)-mediated transcriptional regulation of the human corticotropin-releasing hormone-binding protein promoter: Differential effects of ERalpha and ERbeta. Mol Endocrinol 18: 2908-2923, 2004.

47. Qu N, Zhou XY, Han L, Wang L, Xu JX, Zhang T, Chu J, Chen Q Wang JZ, Zhang Q and Tian Q: Combination of PPT with LiCl treatment prevented bilateral ovariectomy-induced hippocampal-dependent cognition deficit in rats. Mol Neurobiol 53: 894-904, 2016

48. Gujski M,Pinkas J, Wierzbińska-Stępniak A, Owoc A and Bojar I: Does genetic testing for $\mathrm{ER} \alpha$ gene polymorphisms provide new possibilities of treatment for cognitive function disorders in postmenopausal women? Arch Med Sci 13: 1224-1232, 2017. 
49. Witty CF, Foster TC, Semple-Rowland SL and Daniel JM: Increasing hippocampal estrogen receptor alpha levels via viral vectors increases MAP kinase activation and enhances memory in aging rats in the absence of ovarian estrogens. PLOS One 7: e51385, 2012.

50. Ianov L, Kumar A and Foster TC: Epigenetic regulation of estrogen receptor $\alpha$ contributes to age-related differences in transcription across the hippocampal regions CA1 and CA3. Neurobiol Aging 49: 79-85, 2016.

51. Izquierdo I, Furini CR and Myskiw JC: Fear memory. Physiol Rev 96: 695-750, 2016.
52. Sun X,Li X,Pan R, Xu Y, Wang Q and Song M: Total Saikosaponins of Bupleurum yinchowense reduces depressive, anxiety-like behavior and increases synaptic proteins expression in chronic corticosterine-treated mice. BMC Complement Altern Med 18: 117, 2018.

53. Lixing X, Zhouye J, Liting G, Ruyi Z, Rong Q and Shiping M: Saikosaponin-d-mediated downregulation of neurogenesis results in cognitive dysfunction by inhibiting Akt/Foxg-1 pathwayin mice. Toxicol Lett 284: 79-85, 2018.

54. Wang P, Ren J, Tang J, Zhang D, Li B and Li Y: Estrogen-like activities of saikosaponin-d in vitro: A pilot study. Eur J Pharmacol 626: 159-165, 2010. 